\title{
Comportamento sedentário em diferentes domínios de mulheres adultas do sul do Brasil: um estudo de base populacional
}

\author{
Sedentary behavior across different domains among adult women \\ in the south of Brazil: a population based study
}

Cristina Borges Cafruni (https://orcid.org/0000-0002-3568-0785) 1,2

Maria Teresa Anselmo Olinto (https://orcid.org/0000-0002-3950-4594) ${ }^{2}$

Juvenal Soares Dias da Costa (https://orcid.org/0000-0003-3160-6075) ${ }^{2}$

Fernanda Souza de Bairros (https://orcid.org/0000-0002-8611-058X) 1,2

Ruth Liane Henn (https://orcid.org/0000-0002-5056-4934) ${ }^{2}$
${ }^{1}$ Universidade Federal do Rio Grande do Sul. Av. Bento Gonçalves 9500, Agronomia. 91509-900 Porto Alegre RS Brasil. ccafruni@hotmail.com ${ }^{2}$ Universidade do Vale do Rio dos Sinos. São Leopoldo RS Brasil.

\begin{abstract}
The aim of this study was to describe sedentary behavior (SB) across leisure, occupation, and transport domains and determine factors associated with excessive sedentary behavior (ESB). Cross-sectional survey with a representative sample of 1,126 women aged 20-69 years living in São Leopoldo/RS. SB, demographic, socioeconomic, behavioral and health factors data were collected using a questionnaire administered by interviewers. The cut-off point for ESB was defined as the median. Associations were tested using Poisson regression with robust error variance. The medians and interquartile intervals (min/day) for leisure, occupation, and transport $S B$ were 163.9(86.6-2710.5), 51.4(0-257.1), and 17.1(5.737.3), respectively. The likelihood of leisure SB increased with education level, was higher among women who were not employed, lived in household without children, and smokers. In other domains, there was an inverse association between age, being white, economic class, education level, and income and ESB. Direct association between living in a household with a car and excessive transport $S B$ and women who were not employed were $30 \%$ less likely to engage in ESB in this domain. The predominant domain in Total SB was leisure. Associations differed across domains, indicating that domain-specific interventions should be implemented in addressing excessive $S B$.
\end{abstract}

Key words Sedentary lifestyle, Women's health, Epidemiology
Resumo O objetivo deste estudo foi descrever o comportamento sedentário (CS) nos dominios lazer, ocupação e deslocamento e verificar fatores associados ao excesso deste comportamento (ECS). Estudo transversal, com amostra representativa de 1.126 mulheres, 20-69 anos, de São Leopoldo/ RS. CS, variáveis demográficas, socioeconômicas, comportamentais e relacionada à saúde foram avaliados através de questionário, aplicado em forma de entrevista. Considerou-se ECS valores acima da mediana. Utilizou-se regressão de Poisson com variância robusta. As medianas e intervalos interquartílicos (min/dia), para o CS no lazer, ocupação e deslocamento foram, respectivamente, 163,9 (86,6-2710,5), 51,4 (0-257,1) e 17,1 (5,737,3). A probabilidade do ECS no lazer aumentou com a escolaridade, foi maior entre as mulheres que não trabalhavam, sem crianças em casa e fumantes. Nos demais dominios, a probabilidade aumentou inversamente com a idade, foi maior entre mulheres brancas e aumentou com a classe econômica, escolaridade e renda. A probabilidade de ECS no deslocamento também aumentou com o número de carros no domicílio e foi $30 \%$ menor entre mulheres que não trabalhavam. O maior tempo de CS observado foi no dominio do lazer. As associações diferiram segundo o domínio, indicando distintas intervenções.

Palavras-chave Estilo de vida sedentário, Saúde das mulheres, Epidemiologia 


\section{Introdução}

Nas últimas décadas, os estudos que abordaram o estilo de vida das populações e sua relação com a saúde têm destacado a contribuição do comportamento sedentário (CS) como fator de risco para morbimortalidade ${ }^{1,2}$. O CS compreende todas as atividades em posição sentada ou reclinada em que o indivíduo realiza no tempo em que está acordado, envolvendo um gasto energético igual ou inferior a 1,5 Equivalentes Metabólicos (METs) ${ }^{3}$. Este conceito se distingue de inatividade física, quando o indivíduo não atinge a quantidade diária de atividade física $(\mathrm{AF})$ recomendada pelos órgãos de saúde ${ }^{4}$. Evidências produzidas por estudos prospectivos demonstraram que um maior tempo despendido em CS aumenta o risco de diabetes, doenças cardiovasculares, síndrome metabólica e morte ${ }^{5,6}$. Mesmo que altos níveis de AF moderada ( $\sim 60-75 \mathrm{~min} / \mathrm{dia})$ possam eliminar ou atenuar alguns destes riscos ${ }^{7}$, a maioria da população brasileira feminina $(91,5 \%)^{8}$ não alcançou sequer a quantidade recomendada de $\operatorname{AF}(\sim 30 \mathrm{~min} / \mathrm{dia})^{4}$.

De maneira geral, é a soma de todos os comportamentos sedentários realizados durante o dia, ou seja, o CST, que determinará o grau de comprometimento da saúde a que um indivíduo pode estar submetido ${ }^{6}$. Algumas pesquisas, no entanto, sugerem que determinados CS podem se associar mais a algumas morbidades do que outros $^{9,10}$. Se por um lado avaliar o CST auxilia a identificar aqueles indivíduos ou populações com maiores riscos à saúde ${ }^{11}$, este dado sozinho é insuficiente para planejar intervenções, pois não permite conhecer quais são as atividades sedentárias que mais contribuem para o excesso de CST, e em quais contextos elas ocorrem. Nesta perspectiva, tem sido sugerido que as investigações sobre o CS sejam realizadas considerando cada domínio da vida diária, como por exemplo, lazer, deslocamentos e ocupação ${ }^{12}$. Esta abordagem auxilia tanto na compreensão da composição do CST, quanto na investigação dos fatores associados, que podem ser diferentes em cada domínio ${ }^{13,14}$.

No Brasil, poucos estudos epidemiológicos avaliaram o $\mathrm{CS}^{8,15-18} \mathrm{e}$ apenas um analisou de acordo com diferentes domínios ${ }^{16}$. Entretanto, pouco se sabe sobre qual é a contribuição conjunta de fatores demográficos, socioeconômicos, comportamentais e relacionados à saúde na distribuição do CS de populações de países em desenvolvimento. A pesquisa "Condições de Vida e Saúde de Mulheres Adultas: Estudo de Base Populacional no Vale dos Sinos. Avaliação após 10 Anos" oportunizou investigar o CS neste segmento populacional.
Assim, o presente estudo teve como objetivo descrever o CS dessas mulheres nos domínios lazer (CSL), ocupação (CSO) e deslocamento (CSD) e verificar os fatores associados ao excesso de comportamento sedentário (ECS) nos diferentes domínios (ECSL, ECSO e ECSD).

\section{Métodos}

A pesquisa em que este estudo se insere teve delineamento transversal e investigou uma amostra representativa de mulheres de 20 a 69 anos, residentes na zona urbana do município de São Leopoldo, no ano de 2015. Foram excluídas as mulheres grávidas, aquelas sem condições mentais para responder o questionário, de acordo com a observação do entrevistador, e confirmadas por um morador do domicílio, e mulheres impossibilitadas de se locomover, mesmo que parcialmente, na semana anterior à entrevista.

O município de São Leopoldo pertence à região Metropolitana de Porto Alegre, localizando-se a $33 \mathrm{~km}$ da capital do estado do Rio Grande do Sul. Possui uma área de $102.738 \mathrm{~km}^{2}$ e, segundo o Censo 2010, uma população de 214.087 habitantes, com predomínio do sexo feminino $(109.845)^{19}$. A densidade demográfica do município é de 2.060,31 habitantes $/ \mathrm{km}^{2}$. No ano de 2010, o Índice de Desenvolvimento Humano do Município (IDHM) era de 0,739 , classificado como alto e, superior ao índice do Brasil $(0,727)^{20}$.

O cálculo amostral para o estudo maior foi feito para os vários desfechos que seriam investigados na pesquisa, sendo que o maior tamanho foi obtido para o desfecho "exame citopatológico atrasado" e a exposição "escolaridade". O cálculo foi feito considerando-se uma razão de risco de 2,0, nível de 95\% de confiança, poder estatístico de $80 \%$ e razão de não exposto: exposto de 1:2. Ao valor obtido acrescentou-se $10 \%$ para eventuais perdas e recusas e $15 \%$ para controle de fatores de confusão, totalizando 1.281 mulheres. A amostragem foi por conglomerados, em dois estágios. No primeiro estágio, foram sorteados, de forma sistemática, 45 dos 371 setores censitários de São Leopoldo, segundo o censo de $2010^{19}$, e, no segundo estágio, 36 domicílios em cada setor. O percentual de perdas e recusas foi de $12,1 \%$, e a amostra final totalizou 1.126 mulheres entrevistadas. Com base neste número, calculou-se o poder amostral para este estudo. Para avaliar as associações entre as diferentes exposições e os desfechos relativos ao CS do presente estudo, a amostra teve poder de $70 \%$ ou mais para detectar como significativas razões de prevalência de 1,2 
ou maiores, para exposições que afetam de 33,6\% a $78,0 \%$ da população, com um nível de $95 \%$ de confiança.

O comportamento sedentário (CS) foi medido por meio de questionário construído com base na revisão de literatura ${ }^{21,22}$ e de instrumento já existente ${ }^{16}$. A construção do questionário justificou-se por incluir as atividades sedentárias realizadas tanto na postura sentada quanto na posição reclinada, de acordo com a definição de CS, além de registrar atividades nos dias de semana e nos finais de semana. Foram registradas as seguintes situações e/ou locais: a) utilizar computador, $t a-$ blet ou celular para trabalho/estudo em casa; b) utilizar computador, tablet ou celular para lazer em casa; c) assistir à televisão; d) realizar deslocamento motorizado (carro, moto, trem, ônibus); e) no trabalho; f) no colégio, curso ou faculdade; g) beber chimarrão; h) visitar/encontrar os amigos; i) outras atividades (leitura, atividades religiosas e atividades manuais). No caso de duas atividades terem sido realizadas concomitantemente, foi solicitado à respondente relatar somente aquela que considerasse a principal. Para compor o CS total (CST) e dos domínios do lazer (CSL), ocupação (CSO) e deslocamento (CSD), consideraram-se as diferentes situações e locais descritos anteriormente: CST (todas as situações e locais); CSL (b, c, g, h); CSO (a, e, f) e CSD (d). O CST e nos diferentes domínios foram expressos em min/dia. Os valores foram obtidos somandose o tempo gasto em todos os dias da semana e dividindo-se o resultado por 7 . O instrumento de medida do CS teve sua confiabilidade testada por meio do método teste-reteste em uma amostra de 97 participantes do estudo. Os coeficientes de correlação para CST, CSL e CSO foram, respectivamente, $0,79,0,79$ e 0,82 (correlação forte) e para o domínio do deslocamento foi 0,60 (correlação moderada).

Para as variáveis independentes, utilizou-se um questionário padronizado, pré-codificado e pré-testado, aplicado em forma de entrevista. Foram investigadas as variáveis demográficas: idade (categorizada em faixas de 10 anos), cor da pele (branca; não branca), situação conjugal (com companheiro; sem companheiro); socioeconômicas: classe econômica $(\mathrm{A} / \mathrm{B}, \mathrm{C}, \mathrm{D} / \mathrm{E})^{23}$, escolaridade ( 0 a 4,5 a 7,8 a 10,11 a $14, \geq 15$ anos), renda familiar per capita (número de salários mínimos em quartis), trabalho ( tais: fumo (não fumante, fumante), consumo de álcool $(<30 \mathrm{~g} / \mathrm{dia}, \geq 30 \mathrm{~g} / \mathrm{dia})^{24,25}$, AF no lazer $(\geq$ $150 \mathrm{~min} / \mathrm{sem},<150 \mathrm{~min} / \mathrm{sem})^{4}$ e no deslocamento (sim, não), medida por meio de uma adaptação do IPAQ curto (International Physical Activity
Questionnaire $)^{26}$. Na adaptação do IPAQ, visando simplificar o instrumento, foram consideradas somente as atividades de deslocamento e de lazer, sendo que a intensidade das atividades (moderada/vigorosa) foi questionada após o relato da mesma. Além destas variáveis, foram investigadas: carro no domicílio (nenhum, um, dois ou mais), computador no domicílio (nenhum, um, dois ou mais), crianças no domicílio (duas ou mais, uma, nenhuma) e a variável autopercepção de saúde (excelente/muito boa/boa, razoável/ruim).

Todos os entrevistadores foram treinados e participaram do estudo piloto em um setor censitário não sorteado para a pesquisa. A coleta de dados foi realizada em forma de mutirão, durante 8 meses, e sua qualidade foi avaliada em uma amostra aleatória de $10 \%$ das entrevistadas, por meio de algumas questões iguais ao instrumento da pesquisa, não sujeitas à modificação em curto prazo.

O estudo foi aprovado pelo Comitê de Ética em Pesquisa da Universidade do Vale do Rio dos Sinos, e as participantes dos domicílios sorteados só responderam o questionário após a leitura e assinatura do Termo de Consentimento Livre e Esclarecido.

Os dados foram digitados duplamente no programa EpiData versão 3.1, com o objetivo de verificar e corrigir possíveis erros de digitação. A análise descritiva do CST e dos demais domínios foi realizada no software IBM SPSS versão 22.0 (IBM Corp., Armonk, Estados Unidos). O CST e os diferentes domínios foram descritos como medianas e intervalos interquartílicos, uma vez que não tinham distribuição normal. A definição de excesso de CS nos diferentes domínios foi feita utilizando-se a mediana correspondente a cada domínio como ponto de corte. Este procedimento foi adotado em outros estudos como alternativa à falta de um limiar do CS a partir do qual ocorreria risco à saúde ${ }^{16,27}$. Os dados foram expressos como proporção, com seu respectivo intervalo de 95\% de confiança (IC95\%). Como algumas mulheres apresentaram valores irreais de CST (> 24h/dia), arbitrou-se o valor máximo em $1.140 \mathrm{~min} / \mathrm{dia}$, considerando-se um mínimo de 5 h de sono/dia. Valores acima deste ponto de corte foram substituídos pela mediana $(1,1 \%$ da amostra). As associações foram testadas por meio de regressão de Poisson com variância robusta ${ }^{28}$, utilizando-se o software Stata MP versão 14.0 (Stata Corp., College Station, Estados Unidos). As variáveis com $\mathrm{p} \leq 0,20$ na análise bivariada foram levadas para a análise ajustada, a qual foi realizada baseada em um modelo conceitual compreendendo três níveis de determinação. O nível 
1 incluiu as variáveis demográficas, que foram ajustadas entre si, o nível 2 incluiu as variáveis socioeconômicas, que foram ajustadas entre si e pelas variáveis do nível 1 com $p \leq 0,20$, e o nível 3 incluiu, além das variáveis comportamentais, as variáveis carro, criança no domicílio e a variável autopercepção de saúde, as quais foram ajustadas entre si e pelas variáveis dos níveis anteriores com $\mathrm{p} \leq 0,20$. As variáveis situação conjugal, trabalho, carro e criança no domicílio, autopercepção de saúde e as variáveis comportamentais não foram incluídas no modelo que buscou explicar o ECSO, por se entender que estas variáveis não fazem parte do modelo explicativo deste desfecho. Pela mesma razão, as variáveis situação conjugal e computador no domicílio não foram avaliadas para o modelo do ECSD. Foram consideradas associadas ao desfecho as variáveis com $\mathrm{p}<0,05$. Devido ao desenho amostral, as análises foram realizadas utilizando-se o comando svy do Stata.

\section{Resultados}

As entrevistadas apresentaram uma média de idade de 43,3 anos $(\mathrm{DP} \pm 13,4)$ e eram, em sua maioria, brancas $(74,4 \%)$, possuíam companheiro $(63,8 \%)$ e não residiam com criança no domicílio $(59,1 \%)$. Quanto às variáveis socioeconômicas, as mulheres tinham escolaridade média de 9,8 anos (DP $\pm 10,8)$, a maior parte delas trabalhava (56\%), tinha renda per capita $<1,5$ salários mínimos $(74,7 \%)$ e pertencia à classe econômica C $(52,8 \%)$. Do total da amostra, a maioria morava em domicílio que possuía pelo menos um carro $(63,0 \%)$ e um computador $(62,4 \%)$. Destaca-se que grande parte das mulheres não realizou pelo menos $150 \mathrm{~min} / \mathrm{sem}$ de AF no lazer $(85,7 \%)$, por outro lado, não apresentou consumo abusivo de álcool $(97,5 \%)$ ou hábito de fumar $(81,5 \%)$ e relatou sua saúde como boa/muito boa/excelente $(66,3 \%)$ (Tabela 1).

As medianas e intervalos interquartílicos para CST, CSL, CSO e CSD foram, respectivamente $271,4 \mathrm{~min} / \mathrm{dia}(150,0-463,2), 163,9 \mathrm{~min} / \mathrm{dia}(86,8-$ $270,5) ; 51,4 \mathrm{~min} / \mathrm{dia}(0-257,1)$ e $17,1 \mathrm{~min} / \mathrm{dia}$ $(5,7-37,3)$. Já as médias e seus respectivos intervalos de 95\% de confiança (IC95\%) foram: CST - 319,4min/dia $(306,8-331,9)$, CSL - 208,1min/ dia $(197,1-219,2)$, CSO - 141,5min/dia (IC95\%: 128,7-154,4) e CSD - 33,1min/dia (IC95\%: 29,8$36,2)$ (dados não apresentados em tabela).

A contribuição de cada domínio para o CST, na amostra toda e de acordo com as classes econômicas, é apresentada na Figura 1. Para este gráfico, foram utilizados os valores das médias de CS ( $\mathrm{min} / \mathrm{dia})$. Na amostra total, houve um predomínio do CSL (63\%), seguido do CSO (27\%) e do CSD (10\%). Não houve diferença nas distribuições de CSL entre as classes $(p \geq 0,05)$, contudo, estes valores representaram diferentes percentuais na composição do CS total de cada classe. Enquanto que, na classe D/E $82 \%$ do CS total foi composto pelo CSL, este valor correspondeu a $52 \%$ na classe $\mathrm{A} / \mathrm{B}$. A distribuição do $\mathrm{CSO}$ entre as classes econômicas apresentou diferenças significativas $(p<0,001)$, assim como sua contribuição para o CST. O domínio com menor participação no CST (9 a 11\%) foi o CSD, que embora tenha demonstrado proporções semelhantes entre as classes, apresentou diferenças nas suas distribuições $(\mathrm{p}<0,001)$.

Em relação ao ECSL (Tabela 2), após controle para os fatores de confusão, as variáveis que se mantiveram associadas ao desfecho foram: escolaridade, trabalho, crianças no domicílio e fumo. A probabilidade de ECSL foi $30 \%$ maior entre as participantes que tinham 15 ou mais anos de estudo, em comparação àquelas com 0 a 4 anos. Mulheres que não trabalhavam tinham $60 \%$ mais probabilidade de ECSL do que as que trabalhavam. Não ter crianças no domicílio aumentou em $40 \%$ a probabilidade de ECSL. Mulheres fumantes apresentaram uma razão de prevalência (RP) de 1,3 (IC95\%: 1,1-14) de ter ECSL.

Para o ECSO, apenas a variável computador no domicílio perdeu significância estatística na análise ajustada, todas as demais permaneceram significativamente associadas ao desfecho. Observou-se uma associação linear e inversa entre faixa etária e ECSO, enquanto as variáveis classe econômica, escolaridade e renda foram direta e linearmente associadas com o desfecho. Mulheres brancas, em relação às não brancas, eram $40 \%$ mais prováveis de apresentar ECSO (Tabela 3).

Quanto ao ECSD, no modelo ajustado (Tabela 4), as variáveis fumo, AF no lazer, AF no deslocamento e autopercepção de saúde perderam significância estatística, enquanto as demais variáveis mantiveram-se associadas ao desfecho. Quanto menor a idade, maior a probabilidade de ECSD. Mulheres brancas eram 20\% mais prováveis de ter ECSO do que as mulheres não brancas. As variáveis classe econômica, escolaridade e renda associaram-se linearmente e diretamente com ECSO. Mulheres que não trabalhavam apresentaram uma RP de 0,70 (IC95\%: 0,6-08) para ECSO. A probabilidade de ECSD aumentou com o número de carros no domićlíio. 
Tabela 1. Características da amostra do estudo $(\mathrm{n}=$ 1126).

\begin{tabular}{|c|c|c|}
\hline Variável & $\mathbf{n}$ & (\%) \\
\hline \multicolumn{3}{|l|}{ Faixa etária (anos) } \\
\hline 60 a 69 & 163 & 14,5 \\
\hline 50 a 59 & 228 & 20,2 \\
\hline 40 a 49 & 275 & 24,4 \\
\hline 30 a 39 & 244 & 21,7 \\
\hline 20 a 29 & 216 & 19,2 \\
\hline \multicolumn{3}{|l|}{ Cor da pele } \\
\hline Não branca & 288 & 25,6 \\
\hline Branca & 838 & 74,4 \\
\hline \multicolumn{3}{|l|}{ Situação conjugal } \\
\hline Sem companheiro & 408 & 36,2 \\
\hline c/companheiro & 718 & 63,8 \\
\hline \multicolumn{3}{|l|}{ Classe econômica } \\
\hline D e E & 136 & 12,1 \\
\hline $\mathrm{C}$ & 595 & 52,8 \\
\hline A e B & 389 & 34,7 \\
\hline \multicolumn{3}{|l|}{ Escolaridade (anos) } \\
\hline 0 a 4 & 204 & 18,1 \\
\hline 05 a 07 & 253 & 22,5 \\
\hline 08 a 10 & 197 & 17,5 \\
\hline 11 a 14 & 360 & 32,0 \\
\hline 15 anos + & 110 & 9,8 \\
\hline \multicolumn{3}{|l|}{ Renda per capta em SM ${ }^{1}$} \\
\hline Até 0,52 & 276 & 25,3 \\
\hline 0,53 a 0,86 & 270 & 24,8 \\
\hline 0,87 a 1,53 & 271 & 24,9 \\
\hline$\geq 1,54$ & 272 & 25,0 \\
\hline \multicolumn{3}{|l|}{ Trabalho } \\
\hline Sim & 631 & 56,0 \\
\hline Não & 494 & 43,9 \\
\hline \multicolumn{3}{|l|}{ Carro no domicílio } \\
\hline Nenhum & 417 & 37,0 \\
\hline Um & 547 & 48,6 \\
\hline Dois ou mais & 162 & 14,4 \\
\hline \multicolumn{3}{|l|}{ Computador no domicílio } \\
\hline Nenhum & 423 & 37,6 \\
\hline Um & 461 & 40,9 \\
\hline Dois ou mais & 242 & 21,5 \\
\hline \multicolumn{3}{|l|}{ Crianças no domicílio ${ }^{2}$} \\
\hline Duas ou mais & 140 & 12,4 \\
\hline Uma & 320 & 28,4 \\
\hline Nenhuma & 66 & 59,1 \\
\hline \multicolumn{3}{|l|}{ Fumo } \\
\hline Não fumante & 918 & 81,5 \\
\hline Fumante & 208 & 18,5 \\
\hline \multicolumn{3}{|l|}{ Álcool } \\
\hline$<30 \mathrm{~g} /$ dia & 1098 & 97,5 \\
\hline$\geq 30 \mathrm{~g} / \mathrm{dia}$ & 28 & 2,5 \\
\hline \multicolumn{3}{|l|}{ AF no lazer } \\
\hline$\geq 150 \mathrm{~min} / \mathrm{sem}$ & 161 & 14,3 \\
\hline$<150 \mathrm{~min} / \mathrm{sem}$ & 965 & 85,7 \\
\hline \multicolumn{3}{|l|}{ AF no deslocamento } \\
\hline Sim & 744 & 66,1 \\
\hline Não & 382 & 33,9 \\
\hline \multicolumn{3}{|l|}{ Autopercepção de saúde } \\
\hline Excelente/muito boa/boa & 746 & 66,3 \\
\hline Razoável/ruim & 380 & 33,7 \\
\hline Total & 1126 & 100,0 \\
\hline
\end{tabular}

\section{Discussão}

O presente estudo descreveu o CS total e por domínios e verificou os fatores associados ao ECS em cada domínio da vida diária de uma amostra representativa de mulheres adultas de São Leopoldo/RS. Metade da amostra passou cerca de 270 minutos ou mais em CS, ou o equivalente a 4,5 horas por dia, sendo que $63 \%$ do CS total foram destinados ao lazer, 27\% à ocupação e $10 \%$ ao deslocamento. Alguns fatores foram associados aos três domínios estudados, enquanto outros foram domínio-específicos.

Tanto o valor da mediana (271,4min/dia; IIQ: 150,0-463,2) quanto da média (319,4; IC95\%: 306,8-331,9) do CST observados no presente estudo foram semelhantes aos valores encontrados para a população feminina de Pelotas ${ }^{16}$ (mediana: 240,0 $\mathrm{min} / \mathrm{dia}$, IIQ:135,0-480; média: 323,0min/ dia, IC95\%: 305,0-341,0). Já em comparação aos dados de mulheres de Ribeirão Preto ${ }^{18}$ (média: 270,3min/dia; IC95\%: 256,3-284,23), a média de CS das mulheres de São Leopoldo foi cerca de 50 min superior. As comparações, no entanto, são limitadas, uma vez que os instrumentos utilizados para medir o CS diferem entre os estudos ${ }^{12}$. Menores valores de CS entre as mulheres de Ribeirão Preto, por exemplo, podem ser atribuídos, em parte, à utilização do IPAQ, o qual possui uma única questão sobre o tempo sentado, possivelmente subestimando o tempo total ${ }^{18}$. Além disto, o IPAQ não leva em conta o CS despendido nos deslocamentos. O maior tempo de CS observado no presente estudo também poderia ser explicado pelo fato do instrumento utilizado ter avaliado o CS nas posturas sentadas e reclinadas, enquanto os estudos citados verificaram apenas a postura sentada.

Em relação ao CSL, verificou-se que metade das mulheres passaram $163,9 \mathrm{~min} / \mathrm{dia}$ ou mais neste domínio. A comparação deste resultado com dados nacionais não foi possível, pois não foram encontradas pesquisas que avaliaram $o$ CSL da maneira como o do presente estudo, que incluiu outros itens além de assistir à televisão, como o uso de computador para lazer, por exemplo. Embora o hábito de assistir à televisão seja o CSL mais frequente, dados nacionais evidenciaram uma tendência de declínio ao longo dos $\operatorname{anos}^{17}$.

Entre as mulheres que possuíam alguma ocupação $(\mathrm{n}=685)$, metade gastou $50 \mathrm{~min} /$ dia ou mais em CS no trabalho e/ou estudo. Quando se considera os valores médios (141,5min/dia; IC95\%: 121,68-161,41), estes foram semelhantes ao tempo destinado ao trabalho na popula- 


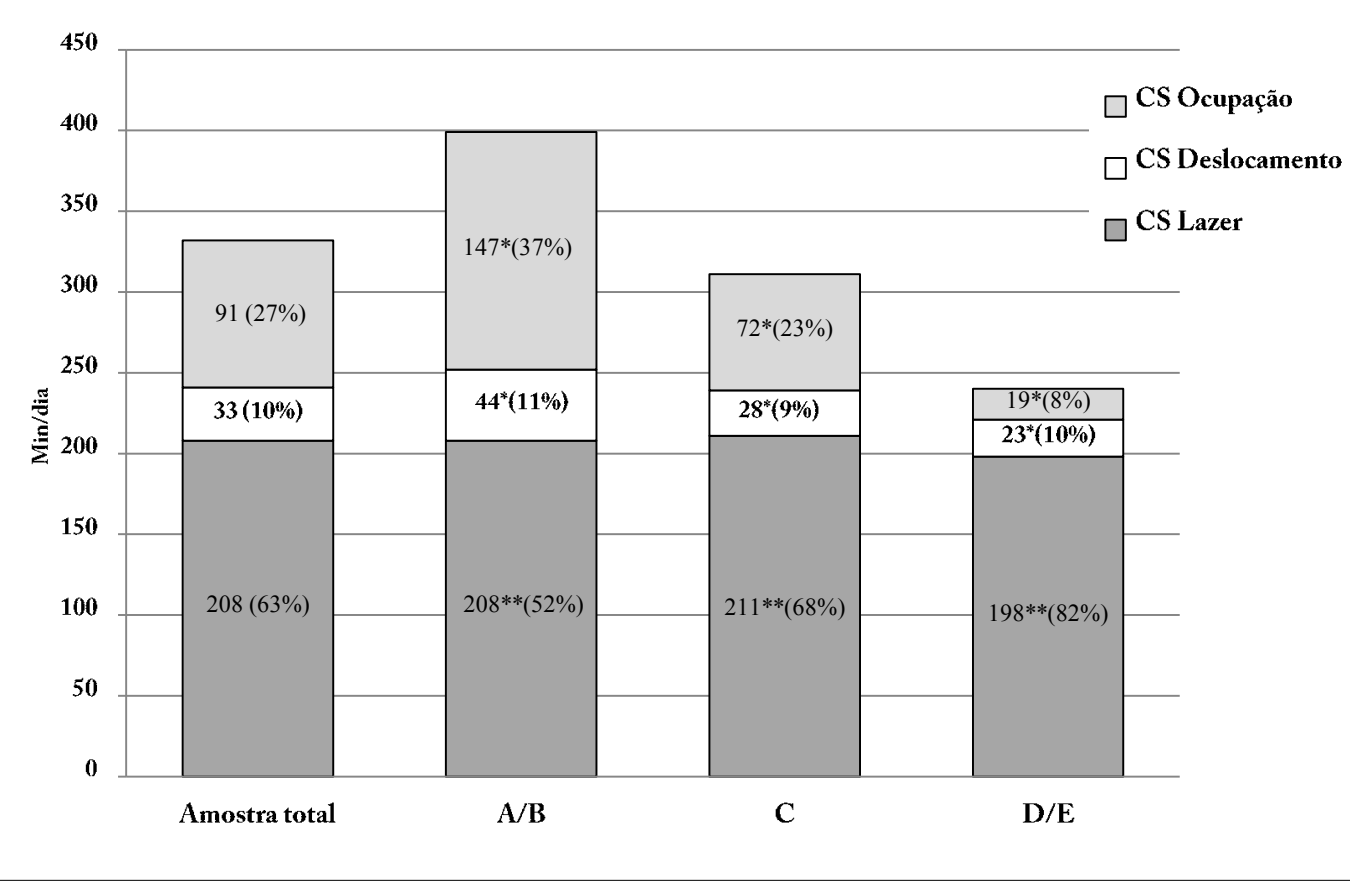

Figura 1. Médias do CS total e dos domínios e contribuição percentual de cada domínio para o total da amostra e segundo a classe econômica

${ }^{\star} \mathrm{p}<0,001 ;{ }^{* *} \mathrm{p}>0,05$ (teste Kruskal-Wallis para diferença entre as distribuições do CS).

ção feminina de Pelotas ( 150min/dia; IC95\%: 130-165) ${ }^{16}$. Já a média de 33min/dia (IC95\%: 29,8-36,2) destinados ao CSD é inferior em aproximadamente $10 \mathrm{~min} / \mathrm{dia}$, comparado aos valores da população feminina de Pelotas ( 45 min/dia; IC95\%: 40-50 $)^{16}$. Para este domínio, foram consideradas todas as mulheres da amostra, visto que os deslocamentos pela cidade podem ocorrer por diferentes razões, tanto para atividades de trabalho e/ou estudo quanto de lazer. Entretanto, a ocupação da mulher pode interferir fortemente em seus deslocamentos diários. Como grande parte da nossa amostra não estava trabalhando à época da pesquisa, isto pode ter contribuído para os menores valores encontrados. Embora não investigadas neste estudo, é provável que as características da cidade de São Leopoldo tenham possibilitado outras formas de deslocamento, como a pé ou de bicicleta, ou tenham reduzido o tempo com transportes motorizados em função dos destinos se encontrarem em localizações mais próximas ${ }^{29,30}$.

$\mathrm{Na}$ análise que avaliou a proporção de cada domínio para compor o CST, considerandose a amostra total, verificou-se que o CSL foi o domínio onde as mulheres despenderam um maior tempo diário (63\% do CST). Este dado é importante, uma vez que o domínio do lazer é o que apresenta maior potencial para ser reduzido $^{31}$, algo desejável se considerarmos o risco aumentado de morbimortalidade associado ao $\mathrm{CS}^{5,6}$. Há evidências, a partir de marcadores de risco cardiometabólicos, de que a substituição de pelo menos 30 minutos diários de CS por AF de intensidades leve ou moderada/intensa traz benefícios para a saúde ${ }^{32}$. No presente estudo, isto corresponderia a uma redução de $20 \%$ no tempo dedicado ao CSL. Outro aspecto em relação aos valores médios de cada domínio do CS e suas respectivas proporções em relação ao CST é que estes podem variar de acordo com a variável de exposição considerada, como demostrado na Figura 1. Optou-se pela variável classe econômica para demonstrar estas diferenças, pois, em tese, as desigualdades socioeconômicas podem influenciar o CS em diferentes situações: em casa, por meio de dispositivos que favorecem a economia de trabalho doméstico e promovem atividades de lazer sedentário (ex. internet, computador); no trabalho, onde as novas tecnologias exigem 
Tabela 2. Prevalência, razões de prevalência (RP) brutas e ajustadas e intervalo de 95\% de confiança (IC95\%) do excesso de comportamento sedentário no lazer de acordo com as características da amostra. $(\mathrm{n}=1126)$.

\begin{tabular}{|c|c|c|c|c|c|}
\hline \multirow{2}{*}{ Variável } & Prevalência & RP bruta & \multirow{2}{*}{ p-valor } & RP ajustada ${ }^{A}$ & \multirow{2}{*}{ p-valor } \\
\hline & \% (IC95\%) & (IC95\%) & & (IC95\%) & \\
\hline \multicolumn{6}{|l|}{ Nível 1} \\
\hline \multicolumn{6}{|l|}{ Faixa etária (anos) } \\
\hline 60 a 69 & $58,9(49,2-68,6)$ & 1 & $0,28^{\star}$ & $\$$ & $\$$ \\
\hline 50 a 59 & $52,6(45,2-60,0)$ & $0,9(0,7-1,1)$ & & & \\
\hline 40 a 49 & $40,0(33,2-46,8)$ & $0,7(0,5-0,8)$ & & & \\
\hline 30 a 39 & $44,3(37,1-51,4)$ & $0,8(0,6-0,9)$ & & & \\
\hline 20 a 29 & $59,7(53,1-66,3)$ & $1,0(0,8-1,2)$ & & & \\
\hline \multicolumn{6}{|l|}{ Cor da pele } \\
\hline Não branca & $48,6(43,1-54,4)$ & 1 & $0,51^{\star *}$ & $\S$ & $\S$ \\
\hline Branca & $50,5(46,5-54,5)$ & $1,0(0,9-1,7)$ & & & \\
\hline Situação conjugal & & & $0,67^{\star \star}$ & & \\
\hline Sem/comp. & $51,0(46,0-56,0)$ & 1 & & $\$$ & $\$$ \\
\hline Com/comp. & $49,4(44,3-54,6)$ & $1,0(0,8-1,1)$ & & & \\
\hline \multicolumn{6}{|l|}{ Nível 2} \\
\hline \multicolumn{6}{|l|}{ Classe econômica } \\
\hline $\mathrm{D}$ e $\mathrm{E}$ & $43,4(37,4-49,4)$ & 1 & $0,13^{*}$ & 1 & \\
\hline $\mathrm{C}$ & $50,4(44,8-56,0)$ & $1,2(1,0-1,4)$ & & $1,2(1,0-1,4)$ & $0,195^{*}$ \\
\hline $\mathrm{A}$ e B & $51,9(46,4-57,4)$ & $1,2(1,0-1,5)$ & & $1,2(0,9-1,5)$ & \\
\hline \multicolumn{6}{|l|}{ Escolaridade (anos) } \\
\hline 0 a 4 & $48,5(42,0-55,1)$ & 1 & $0,13^{*}$ & 1 & \\
\hline 05 a 07 & $47,4(41,6-53,2)$ & $1,0(0,8-1,2)$ & & $1,0(0,9-1,2)$ & $0,040^{*}$ \\
\hline 08 a 10 & $50,3(42,0-58,6)$ & $1,0(0,9-1,3)$ & & $1,1(0,9-1,4)$ & \\
\hline 11 a 14 & $51,1(45,4-56,7)$ & $1,1(0,9-1,2)$ & & $1,1(0,9-1,3)$ & \\
\hline 15 & $54,5(46,0-63,1)$ & $1,1(0,9-1,4)$ & & $1,3(1,1-1,7)$ & \\
\hline \multicolumn{6}{|l|}{ Renda em SM ${ }^{1}$} \\
\hline Até 0,52 & $47,5(41,4-53,5)$ & 1 & $0,21^{\star}$ & $\S$ & $\S$ \\
\hline 0,53 a 0,86 & $48,5(42,0-55,0)$ & $1,0(0,9-1,2)$ & & & \\
\hline 0,87 a 1,53 & $51,3(43,7-58,9)$ & $1,1(0,9-1,3)$ & & & \\
\hline$\geq 1,54$ & $52,9(46,0-59,9)$ & $1,1(0,9-1,3)$ & & & \\
\hline \multicolumn{6}{|l|}{ Trabalho } \\
\hline Sim & $40,6(36,0-45,2)$ & 1 & $<0,001^{\star *}$ & 1 & $<0,001^{* *}$ \\
\hline Não & $61,9(56,8-67,0)$ & $1,5(1,3-1,8)$ & & $1,6(1,4-1,9)$ & \\
\hline
\end{tabular}

atividades laborais na posição sentada; e nos deslocamentos, através do acesso ao transporte automotivo ${ }^{33}$. Apesar de existirem diferenças metodológicas na avaliação do CS, os resultados foram semelhantes aos dados de Pelotas ${ }^{16}$, que mostrou uma diminuição da participação do CS no domínio da ocupação e aumento da proporção do CSL à medida que piorou a classe econômica, e uma estabilidade na proporção de CSD entre as três classes econômicas. Uma possível explicação para esta diferença entre as proporções dos domínios do lazer e do trabalho entre as classes econômicas, é que as mulheres pertencentes às classes mais baixas devem trabalhar em ocupações com maiores exigências físicas, e, consequentemente, despender uma menor quantidade de tempo na postura sentada. No entanto, apesar da marcante diferença entre as proporções de CSL entre as classes econômicas, em nosso estudo, não houve diferença significativa nas distribuições de CSL entre as classes. Estes dados são importantes, pois revelam que as intervenções podem ser dirigidas para um determinado domínio ${ }^{34}$, de acordo com as características da população estudada.

$\mathrm{Na}$ análise ajustada para o ECSL, verificouse uma associação linear e direta da escolaridade com este domínio. Este achado é consistente com a literatura que considerou atividades de 
Tabela 2. Prevalência, razões de prevalência (RP) brutas e ajustadas e intervalo de 95\% de confiança (IC95\%) do excesso de comportamento sedentário no lazer de acordo com as características da amostra. $(\mathrm{n}=1126)$.

\begin{tabular}{|c|c|c|c|c|c|}
\hline Variável & $\begin{array}{c}\text { Prevalência } \\
\%(\text { IC95\%) }\end{array}$ & $\begin{array}{c}\text { RP bruta } \\
\text { (IC95\%) }\end{array}$ & p-valor & $\frac{\text { RP ajustada }}{\text { A }}$ & p-valor \\
\hline \multicolumn{6}{|l|}{ Nível 3} \\
\hline \multicolumn{6}{|l|}{ Carro no domicílio } \\
\hline Nenhum & $50,1(45,5-54,0)$ & 1 & $0,91^{\star}$ & $\$$ & $\$$ \\
\hline Um & $50,1(44,8-55,3)$ & $1,0(0,9-1,1)$ & & & \\
\hline Dois ou mais & $49,4(39,8-58,9)$ & $1,0(0,8-1,2)$ & & & \\
\hline \multicolumn{6}{|l|}{ Computador no domicílio } \\
\hline Nenhum & $47,3(42,8-51,7)$ & 1 & $0,11^{\star}$ & 1 & $0,325^{\star}$ \\
\hline $\mathrm{Um}$ & $50,1(44,7-55,5)$ & $1,1(0,9-1,2)$ & & $1,0(0,9-1,2)$ & \\
\hline Dois ou mais & $54,5(47,1-62,0)$ & $1,5(1,0-1,4)$ & & $1,1(0,9-1,4)$ & \\
\hline \multicolumn{6}{|l|}{ Criança no domicílio ${ }^{2}$} \\
\hline Duas ou mais & $41,4(30,3-52,5)$ & 1 & $<0,001^{\star}$ & 1 & $0,001^{\star}$ \\
\hline Uma & $40,6(34,4-46,8)$ & $1,0(0,7-1,4)$ & & $1,0(0,7-1,4)$ & \\
\hline Nenhuma & $56,3(52,0-60,6)$ & $1,4(1,0-1,8)$ & & $1,4(1,0-1,8)$ & \\
\hline \multicolumn{6}{|l|}{ Fumo } \\
\hline Não fumante & $48,5(44,5-52,5)$ & 1 & $<0,001$ & 1 & $<0,001^{\star *}$ \\
\hline Fumante & $56,7(50,9-62,6)$ & $1,2(1,0-1,3)$ & & $1,3(1,1-1,4)$ & \\
\hline \multicolumn{6}{|l|}{ Álcool } \\
\hline$<30 \mathrm{~g} / \mathrm{dia}$ & $49,7(46,0-53,5)$ & 1 & $0,23^{\star *}$ & $\S$ & $\S$ \\
\hline$\geq 30 \mathrm{~g} / \mathrm{dia}$ & $60,7(41,2-80,2)$ & $1,2(0,9-1,7)$ & & & \\
\hline \multicolumn{6}{|l|}{ AF no lazer } \\
\hline$\geq 150 \mathrm{~min} / \mathrm{sem}$ & $52,6(45,7-59,5)$ & 1 & $0,30^{\star *}$ & $\S$ & $\$$ \\
\hline$<150 \mathrm{~min} / \mathrm{sem}$ & $49,2(45,5-52,9)$ & $0,9(0,8-1,1)$ & & & \\
\hline \multicolumn{6}{|l|}{ AF no deslocamento } \\
\hline Sim & $49,3(44,9-53,7)$ & 1 & $0,49^{\star *}$ & $\$$ & $\S$ \\
\hline Não & $51,3(46,2-56,4)$ & $1,0(0,9-1,2)$ & & & \\
\hline \multicolumn{6}{|l|}{ Autopercepção de saúde } \\
\hline Excelente/muito boa/boa & $48,3(44,1-52,4)$ & 1 & $0,03^{\star *}$ & 1 & $0,489^{\star *}$ \\
\hline Razoável/ruim & $53,4(48,9-58,0)$ & $1,1(1,0-1,2)$ & & $1,0(0,9-1,1)$ & \\
\hline Total & $50,0(46,3-53,7)$ & & & & \\
\hline
\end{tabular}

lazer sedentárias além do tempo assistindo à televisão ${ }^{27,35}$, como no presente estudo. Assim, é provável que as mulheres com maior escolaridade realizavam outras formas de lazer sedentário. Observou-se, também, uma associação positiva do ECSL com não estar trabalhando e com menor número de crianças no domicílio, o que pode indicar que mulheres com mais tempo livre gastam esse tempo em atividades de lazer sedentárias $^{36}$. No presente estudo, mulheres fumantes apresentaram maior probabilidade de ECSL, sugerindo a ocorrência de um cluster de comportamentos não saudáveis ${ }^{37}$. Embora ainda não haja evidências sobre quais os tipos de intervenções são mais eficazes para diminuir o $\mathrm{ECSL}^{38}$, os dados obtidos com este estudo sugerem que elas podem ser realizadas com base na promoção de um estilo de vida saudável, e também através da conscientização sobre a importância de se evitar um tempo excessivo de $\mathrm{CS}^{39,40}$.

Para o ECSO, encontrou-se uma associação linear e inversa com a idade, um achado de difícil explicação. No presente estudo, esta associação poderia ser explicada, em parte, pelo fato do CSO ter considerado também o tempo sentado para estudar, o qual é naturalmente maior em mulheres mais jovens, entretanto, estudos que avaliaram somente o CS no trabalho encontraram resultado semelhante ${ }^{41}$. Outra variável demográfica que permaneceu no modelo final foi a cor da pele, evidenciando que mulheres brancas apresentaram uma probabilidade 38\% 
Tabela 3. Razões de prevalência (RP) brutas e ajustadas e intervalo de 95\% de confiança (IC95\%) do excesso de comportamento sedentário na ocupação de acordo com as características da amostra. $(\mathrm{n}=685)$.

\begin{tabular}{|c|c|c|c|c|c|}
\hline \multirow{2}{*}{ Variável } & Prevalência & RP bruta & \multirow{2}{*}{ p-valor } & RP ajustada ${ }^{A}$ & \multirow{2}{*}{ p-valor } \\
\hline & \% (IC95\%) & (IC95\%) & & (IC95\%) & \\
\hline \multicolumn{6}{|l|}{ Nível 1} \\
\hline \multicolumn{6}{|l|}{ Faixa etária (anos) } \\
\hline 60 a 69 & $42,9(26,2-59,5)$ & 1 & $<0,001^{\star}$ & 1 & $<0,001^{\star}$ \\
\hline 50 a 59 & $35,7(24,8-46,7)$ & $0,8(0,5-1,3)$ & & $0,8(0,5-1,3)$ & \\
\hline 40 a 49 & $45,2(38,3-52,2)$ & $1,1(0,7-1,5)$ & & $1,1(0,7-1,5)$ & \\
\hline 30 a 39 & $51,7(44,0-59,5)$ & $1,2(0,8-1,8)$ & & $1,2(0,8-1,8)$ & \\
\hline 20 a 29 & $66,0(57,3-74,6)$ & $1,5(1,0-2,3)$ & & $1,5(1,0-2,4)$ & \\
\hline \multicolumn{6}{|l|}{ Cor da pele } \\
\hline Não branca & $38,3(31,9-44,8)$ & 1 & $<0,001^{\star}$ & 1 & $<0,001^{\star *}$ \\
\hline Branca & $52,9(47,1-58,7)$ & $1,4(1,2-1,6)$ & & $1,4(1,2-1,6)$ & \\
\hline \multicolumn{6}{|l|}{ Situação conjugal } \\
\hline Sem/comp. & $50,4(43,1-57,6)$ & NA & & NA & \\
\hline Com/comp. & $48,7(43,3-54,1)$ & & & & \\
\hline \multicolumn{6}{|l|}{ Nível 2} \\
\hline \multicolumn{6}{|l|}{ Classe econômica } \\
\hline $\mathrm{D}$ e E & $19,4(12,1-26,7)$ & 1 & $<0,001^{\star}$ & 1 & $0,033^{*}$ \\
\hline $\mathrm{C}$ & $41,8(35,8-47,8)$ & $2,2(1,5-3,2)$ & & $1,4(0,9-2,1)$ & \\
\hline A e B & $66,2(60,2-72,1)$ & $3,4(2,3-5,1)$ & & $1,6(1,0-2,6)$ & \\
\hline \multicolumn{6}{|l|}{ Escolaridade (anos) } \\
\hline 0 a 4 & $24,7(15,9-33,5)$ & 1 & $<0,001^{\star}$ & 1 & $<0,001^{\star}$ \\
\hline 05 a 07 & $25,5(18,2-32,9)$ & $1,0(0,7-1,6)$ & & $0,9(0,6-1,4)$ & \\
\hline 08 a 10 & $40,0(31,4-48,6)$ & $1,6(1,0-2,7)$ & & $1,3(0,8-2,1)$ & \\
\hline 11 a 14 & $65,2(59,1-71,3)$ & $2,6(1,8-3,9)$ & & $1,7(1,1-2,5)$ & \\
\hline 15 & $78,0(69,8-86,2)$ & $3,2(2,2-4,5)$ & & $1,7(1,1-2,6)$ & \\
\hline \multicolumn{6}{|l|}{ Renda em SM ${ }^{1}$} \\
\hline Até 0,52 & $28,4(22,5-34,2)$ & 1 & $<0,001^{\star}$ & 1 & $<0,001^{\star}$ \\
\hline 0,53 a 0,86 & $36,2(30,2-42,3)$ & $1,3(1,0-1,7)$ & & $1,2(0,9-1,6)$ & \\
\hline 0,87 a 1,53 & $51,6(44,1-59,2)$ & $1,8(1,4-2,3)$ & & $1,4(1,0-1,8)$ & \\
\hline$\geq 1,54$ & $71,5(64,5-78,5)$ & $2,5(1,9-3,2)$ & & $1,7(1,3-2,2)$ & \\
\hline \multicolumn{6}{|l|}{ Trabalho } \\
\hline Sim & $51,3(45,8-56,9)$ & NA & & NA & \\
\hline Não & $25,9(14,7-37,1)$ & & & & \\
\hline
\end{tabular}

maior de ECSO comparadas com as mulheres não brancas. Um estudo que avaliou diferenças ocupacionais segundo a cor da pele mostrou que mulheres brancas têm maior chance de trabalhar em atividades administrativas ou técnicas do que as mulheres não brancas ${ }^{42}$. Independente da cor da pele, as variáveis socioeconômicas analisadas foram diretamente associadas com ECSO, demonstrando consistência com a literatura ${ }^{41,43}$. Embora não tenha sido avaliado o tipo de ocupação, estudos observaram que algumas categorias de trabalho relacionadas com a administração, escritórios e serviços, elevaram a chance dos trabalhadores apresentarem altos níveis de CS comparadas com ocupações com maiores exigências físicas ${ }^{44,45}$. Estes resultados indicam que as mulheres com ECSO se beneficiariam de modificações realizadas no ambiente laboral, como a utilização de mesas que permitem alternar as posturas de trabalho ${ }^{46}$.

$\mathrm{Na}$ análise ajustada para o ECSD, todas as variáveis sociodemográficas analisadas foram associadas ao desfecho e a direção destas associações foi a mesma observada para o ECSO. Outras variáveis que no modelo final permaneceram associadas diretamente com ECSD foram o trabalho e a presença de carro no domicílio. Para este domínio, não encontramos estudos com mode- 
Tabela 3. Razões de prevalência (RP) brutas e ajustadas e intervalo de 95\% de confiança (IC95\%) do excesso de comportamento sedentário na ocupação de acordo com as características da amostra. ( $\mathrm{n}=685)$.

\begin{tabular}{|c|c|c|c|c|c|}
\hline \multirow{2}{*}{ Variável } & \multirow{2}{*}{$\begin{array}{l}\text { Prevalência } \\
\text { \% (IC95\%) } \\
\end{array}$} & \multirow{2}{*}{$\begin{array}{l}\text { RP bruta } \\
\text { (IC95\%) }\end{array}$} & \multirow{2}{*}{ p-valor } & \multirow{2}{*}{$\begin{array}{c}\text { RP ajustada }^{\mathrm{A}} \\
(\mathrm{IC} 95 \%)\end{array}$} & \multirow{2}{*}{ p-valor } \\
\hline & & & & & \\
\hline \multicolumn{6}{|l|}{ Nível 3} \\
\hline \multicolumn{6}{|l|}{ Carro no domicílio } \\
\hline Nenhum & $36,3(29,9-42,7)$ & NA & & NA & \\
\hline Um & $53,2(47,2-59,3)$ & & & & \\
\hline Dois ou mais & $63,6(53,7-73,4)$ & & & & \\
\hline \multicolumn{6}{|l|}{ Computador no domicílio } \\
\hline Nenhum & $31,4(25,4-37,5)$ & 1 & $<0,001^{\star}$ & 1 & $0,338^{\star}$ \\
\hline Um & $51,4(44,3-58,5)$ & $1,6(1,3-2,1)$ & & $1,1(0,8-1,4)$ & \\
\hline Dois ou mais & $69,4(62,3-76,4)$ & $2,2(1,8-2,7)$ & & $1,1(0,9-1,5)$ & \\
\hline \multicolumn{6}{|l|}{ Criança no domicílio ${ }^{2}$} \\
\hline Duas ou mais & $33,7(22,6-44,9)$ & NA & & NA & \\
\hline Uma & $45,7(39,6-51,8)$ & & & & \\
\hline Nenhuma & $54,6(47,3-61,8)$ & & & & \\
\hline \multicolumn{6}{|l|}{ Fumo } \\
\hline Não fumante & $52,3(46,7-57,9)$ & NA & & NA & \\
\hline Fumante & $35,8(28,4-43,2)$ & & & & \\
\hline \multicolumn{6}{|l|}{ Álcool } \\
\hline$<30$ g/dia & $49,5(44,1-54,9)$ & NA & & $\mathrm{NA}$ & \\
\hline$\geq 30 \mathrm{~g} / \mathrm{dia}$ & $44,4(22,8-66,1)$ & & & & \\
\hline \multicolumn{6}{|l|}{ AF no lazer } \\
\hline$\geq 150 \mathrm{~min} / \mathrm{sem}$ & $66,3(57,4-75,0)$ & NA & & NA & \\
\hline$<150 \mathrm{~min} / \mathrm{sem}$ & $43,8(38,8-48,8)$ & & & & \\
\hline \multicolumn{6}{|l|}{ AF no deslocamento } \\
\hline $\operatorname{Sim}$ & $46,1(40,7-51,5)$ & NA & & NA & \\
\hline Não & $55,1(46,1-64,0)$ & & & & \\
\hline \multicolumn{6}{|l|}{ Autopercepção de saúde } \\
\hline Excelente/muito boa/boa & $54,0(48,4-59,6)$ & NA & & NA & \\
\hline Razoável/ruim & $35,3(28,1-42,5)$ & & & & \\
\hline Total & $49,3(44,0-54,7)$ & & & & \\
\hline
\end{tabular}

los explicativos exclusivamente para populações femininas, o que constitui um limitador para a discussão dos nossos resultados. Uma questão que necessita maior investigação é verificar se um ambiente com condições mais favoráveis para caminhar ou pedalar poderia diminuir o ECSD entre as mulheres ${ }^{47}$.

Entre as vantagens da presente pesquisa, destacamos tratar-se de um estudo de base populacional, onde os resultados podem ser extrapolados para a população feminina de São Leopoldo. O CS foi medido através de várias atividades, considerando-se todos os dias da semana e do final de semana. Além disto, nossa análise foi realizada para três domínios de CS, e levou em conta diversos fatores de exposição.
Algumas limitações, entretanto, devem ser consideradas. Primeiramente, por ser um estudo com delineamento transversal, não se pode descartar a presença de causalidade reversa. Outra limitação diz respeito ao questionário utilizado, o qual ainda não teve sua validade testada. Assim, não se sabe se as mulheres superestimaram ou subestimaram o CS. Também, é possível que alguns CS realizados concomitantemente tenham sido relatados duplamente, superestimando o tempo do CST. Por fim, apesar da utilização da mediana auxiliar na caracterização das mulheres com ECS, estes valores podem não representar riscos à saúde. Na literatura, há evidências de que o risco de mortalidade por todas as causas, em adultos, aumenta a partir de $7 \mathrm{~h}$ diárias de CST, medido 
Tabela 4. Prevalência, razões de prevalências (RP) brutas e ajustadas e intervalo de 95\% de confiança (IC95\%) do excesso de comportamento sedentário no deslocamento de acordo com as características da amostra $(\mathrm{n}=1126)$.

\begin{tabular}{|c|c|c|c|c|c|}
\hline \multirow{2}{*}{ Variável } & Prevalência & RP bruta & \multirow{2}{*}{ p-valor } & RP ajustada ${ }^{A}$ & \multirow{2}{*}{ p-valor } \\
\hline & \% (IC95\%) & (IC95\%) & & (IC95\%) & \\
\hline \multicolumn{6}{|l|}{ Nível 1} \\
\hline \multicolumn{6}{|l|}{ Faixa etária (anos) } \\
\hline 60 a 69 & $37,4(30,3-44,6)$ & 1 & $<0,001^{\star}$ & 1 & $<0,001^{\star}$ \\
\hline 50 a 59 & $38,6(31,2-46,0)$ & $1,0(0,8-1,3)$ & & $1,0(0,8-1,3)$ & \\
\hline 40 a 49 & $53,5(47,1-59,8)$ & $1,4(1,2-1,7)$ & & $1,4(1,2-1,8)$ & \\
\hline 30 a 39 & $54,1(46,9-61,3)$ & $1,5(1,2-1,8)$ & & $1,4(1,2-1,8)$ & \\
\hline 20 a 29 & $57,4(51,4-63,4)$ & $1,5(1,3-1,9)$ & & $1,5(1,3-1,9)$ & \\
\hline \multicolumn{6}{|l|}{ Cor da pele } \\
\hline Não branca & $43,1(36,6-49,5)$ & 1 & $0,064^{\star \star}$ & 1 & $0,046^{* *}$ \\
\hline Branca & $51,1(45,9-56,2)$ & $1,2(1,0-1,4)$ & & $1,2(1,0-1,4)$ & \\
\hline \multicolumn{6}{|l|}{ Situação conjugal } \\
\hline Sem/comp. & $48,0(43,2-52,9)$ & NA & & NA & \\
\hline Com/comp. & $49,6(44,6-54,6)$ & & & & \\
\hline \multicolumn{6}{|l|}{ Nível 2} \\
\hline \multicolumn{6}{|l|}{ Classe econômica } \\
\hline $\mathrm{D}$ e $\mathrm{E}$ & $5,7(0,0-19,0)$ & 1 & $<0,001^{\star}$ & 1 & $0,018^{*}$ \\
\hline $\mathrm{C}$ & $14,3(5,0-30,7)$ & $1,7(1,3-2,3)$ & & $1,3(1,0-1,8)$ & \\
\hline A e B & $28,6(12,9-55,7)$ & $2,5(1,9-3,4)$ & & $1,5(1,0-2,1)$ & \\
\hline \multicolumn{6}{|l|}{ Escolaridade (anos) } \\
\hline 0 a 4 & $27,0(21,2-32,7)$ & 1 & $<0,001^{\star}$ & 1 & $<0,001^{\star}$ \\
\hline 05 a 07 & $39,5(33,7-45,3)$ & $1,5(1,1-1,9)$ & & $1,2(0,9-1,7)$ & \\
\hline 08 a 10 & $40,6(32,0-49,2)$ & $1,5(1,1-2,0)$ & & $1,2(0,9-1,6)$ & \\
\hline 11 a 14 & $64,4(58,8-70,2)$ & $2,4(1,9-3,0)$ & & $1,6(1,2-2,1)$ & \\
\hline 15 & $77,3(69,2-85,4)$ & $2,9(2,3-3,6)$ & & $1,6(1,2-2,2)$ & \\
\hline \multicolumn{6}{|l|}{ Renda em SM ${ }^{1}$} \\
\hline Até 0,52 & $33,0(28,0-38,0)$ & 1 & $<0,001^{\star}$ & 1 & $0,006^{*}$ \\
\hline 0,53 a 0,86 & $44,1(45,1-60,4)$ & $1,3(1,1-1,6)$ & & $1,2(1,0-1,5)$ & \\
\hline 0,87 a 1,53 & $52,8(45,1-60,4)$ & $1,6(1,3-1,9)$ & & $1,2(1,0-1,5)$ & \\
\hline$\geq 1,54$ & $68,0(60,7-75,3)$ & $2,1(1,7-2,5)$ & & $1,4(1,1-1,7)$ & \\
\hline \multicolumn{6}{|l|}{ Trabalho } \\
\hline Sim & $61,2(56,4-66,0)$ & 1 & $<0,001^{\star}$ & 1 & $<0,001^{*}$ \\
\hline Não & $33,6(29,2-38,0)$ & $0,6(0,5-0,6)$ & & $0,7(0,6-0,8)$ & \\
\hline
\end{tabular}

continua

através de questionário ${ }^{48}$. De acordo com este dado, um quarto da amostra do presente estudo teria um risco aumentado de mortalidade, pois apresentou, no mínimo, 7,7h/dia de CST. Assim, a utilização da mediana poderia atenuar as medidas de efeito. No entanto, ainda não existem critérios para classificar o ECS em cada domínio.

Neste estudo, o CSL foi o domínio predominante do CST. As proporções e as distribuições do CS variaram de acordo com a classe econômica. Demostrou-se que variáveis demográficas se associaram ao ESCO e ECSD, mas não ao ECSL. Em todos os domínios, pelo menos uma variá- 
Tabela 4. Prevalência, razões de prevalências (RP) brutas e ajustadas e intervalo de 95\% de confiança (IC95\%) do excesso de comportamento sedentário no deslocamento de acordo com as características da amostra $(\mathrm{n}=1126)$.

\begin{tabular}{|c|c|c|c|c|c|}
\hline \multirow{2}{*}{ Variável } & Prevalência & RP bruta & \multirow{2}{*}{ p-valor } & RP ajustada ${ }^{A}$ & \multirow{2}{*}{ p-valor } \\
\hline & $\%(\mathrm{IC} 95 \%)$ & (IC95\%) & & (IC95\%) & \\
\hline \multicolumn{6}{|l|}{ Nível 3} \\
\hline \multicolumn{6}{|l|}{ Carro no domicílio } \\
\hline Nenhum & $34,3(29,6-39,0)$ & 1 & $<0,001^{\star}$ & 1 & $<0,001^{*}$ \\
\hline Um & $53,4(48,7-58,1)$ & $1,6(1,3-1,8)$ & & $1,2(1,0-1,5)$ & \\
\hline Dois ou mais & $72,2(62,2-82,3)$ & $2,1(1,8-2,5)$ & & $1,5(1,2-1,8)$ & \\
\hline \multicolumn{6}{|l|}{ Computador no domicílio } \\
\hline Nenhum & $36,2(31,7-40,7)$ & NA & & NA & \\
\hline Um & $49,7(45,0-54,4)$ & & & & \\
\hline Dois ou mais & $70,2(61,7-78,8)$ & & & & \\
\hline \multicolumn{6}{|l|}{ Criança no domicílio ${ }^{2}$} \\
\hline Duas ou mais & $43,6(32,7-54,4)$ & 1 & $0,751^{\star}$ & $\S$ & $\S$ \\
\hline Uma & $52,5(47,5-57,5)$ & $1,2(0,9-1,6)$ & & & \\
\hline Nenhuma & $48,5(43,4-53,6)$ & $1,1(0,9-1,4)$ & & & \\
\hline \multicolumn{6}{|l|}{ Fumo } \\
\hline Não fumante & $51,0(46,2-55,9)$ & 1 & $0,016^{\star *}$ & 1 & $0,738^{\star *}$ \\
\hline Fumante & $40,4(33,9-46,8)$ & $0,8(0,7-1,0)$ & & $1,0(0,8-1,1)$ & \\
\hline \multicolumn{6}{|l|}{ Álcool } \\
\hline$<30$ g/dia & $48,8(44,6-53,1)$ & 1 & $0,227^{\star *}$ & $\S$ & $\S$ \\
\hline$\geq 30 \mathrm{~g} / \mathrm{dia}$ & $57,1(42,6-71,7)$ & $1,2(0,9-1,5)$ & & & \\
\hline \multicolumn{6}{|l|}{ AF no lazer } \\
\hline$\geq 150 \mathrm{~min} / \mathrm{sem}$ & $56,3(49,6-63,1)$ & 1 & $0,002^{\star *}$ & 1 & $0,697^{\star *}$ \\
\hline$<150 \mathrm{~min} / \mathrm{sem}$ & $46,7(42,6-50,8)$ & $0,8(0,7-0,9)$ & & $1,0(0,9-1,1)$ & \\
\hline \multicolumn{6}{|l|}{ AF no deslocamento } \\
\hline Sim & $45,8(41,9-49,7)$ & 1 & $0,008^{\star *}$ & 1 & $0,536^{\star *}$ \\
\hline Não & $55,2(47,9-62,6)$ & $1,2(1,1-1,34)$ & & $1,0(0,9-1,1)$ & \\
\hline \multicolumn{6}{|l|}{ Autopercepção de saúde } \\
\hline Excelente/muito boa/boa & $55,0(49,6-60,3)$ & 1 & $<0,001^{\star *}$ & 1 & $0,668^{\star *}$ \\
\hline Razoável/ruim & $37,4(33,4-41,4)$ & $0,7(0,6-0,8)$ & & $1,0(0,9-1,1)$ & \\
\hline Total & $49,0(44,8-53,2)$ & & & & \\
\hline
\end{tabular}

dem auxiliar na definição de intervenções para diminuir o CS em cada domínio. Para ampliar a compreensão dos fatores associados ao CS, futuros estudos devem incluir variáveis sociais, políticas e ambientais na análise.

\section{Colaboradores}

CB Cafruni revisou a literatura, elaborou o projeto e o instrumento de coleta de dados, realizou treinamento dos entrevistadores, supervisionou o trabalho de campo, trabalhou na análise dos dados e na redação do artigo. MTA Olinto coordenou a pesquisa e contribuiu na redação do artigo. JSD Costa coordenou a pesquisa e contribuiu na redação do artigo. FS Bairros contribuiu na elaboração do projeto e na construção do instrumento de coleta de dados, realizou treinamento das entrevistadoras, coordenou o trabalho de campo, trabalhou na redação do artigo. RL Henn elaborou o projeto e o instrumento de coleta de dados, trabalhou na análise dos dados e na redação do artigo. 


\section{Referências}

1. Dunstan DW, Barr EL, Healy GN, Salmon J, Shaw JE, Balkau B, Magliano DJ, Cameron AJ, Zimmet PZ, Owen N. Television viewing time and mortality: the Australian Diabetes, Obesity and Lifestyle Study (AusDiab). Circulation 2010; 121(3):384-391.

2. Owen N, Healy GN, Matthews CE, Dunstan DW. Too much sitting: the population health science of sedentary behavior. Exerc Sport Sci Rev 2010; 38(3):105-113.

3. Tremblay MS, Aubert S, Barnes JD, Saunders TJ, Carson V, Latimer-Cheung AE, Chastin SFM, Altenburg TM, Chinapaw MJM, SBRN Terminology Consensus Project Participants. Sedentary Behavior Research Network (SBRN) - Terminology Consensus Project process and outcome. Int J Behav Nutr Phys Act 2017; 14(1):75.

4. World Health Organization (WHO). Global recommendations on physical activity for health. Geneva: WHO; 2010

5. Wilmot EG, Edwardson CL, Achana FA, Davies MJ, Gorely T, Gray LJ, Khunti K, Yates T, Biddle SJ. Sedentary time in adults and the association with diabetes, cardiovascular disease and death: systematic review and meta-analysis. Diabetologia 2012; 55(11):28952905.

6. Ford ES, Caspersen CJ. Sedentary behaviour and cardiovascular disease: a review of prospective studies. Int J Epidemiol 2012; 41(5):1338-1353.

7. Ekelund U, Steene-Johannessen J, Brown WJ, Fagerland MW, Owen N, Powell KE, Bauman A, Lee IM, Lancet Physical Activity Series 2 Executive Committee, Lancet Sedentary Behaviour Working Group. Does physical activity attenuate, or even eliminate, the detrimental association of sitting time with mortality? A harmonised meta-analysis of data from more than 1 million men and women. Lancet 2016; 388(10051):1302-1310.

8. Knuth AG, Malta DC, Dumith SC, Pereira CA, Morais Neto OL, Temporao JG, Penna G, Hallal PC. Practice of physical activity and sedentarism among Brazilians: results of the National Household Sample Survey-2008. Cien Saude Colet 2011; 16(9):3697-3705.

9. Saidj M, Jorgensen T, Jacobsen RK, Linneberg A, Aadahl M. Separate and joint associations of occupational and leisure-time sitting with cardio-metabolic risk factors in working adults: a cross-sectional study. PLoS One 2013; 8(8):e70213.

10. Hsueh MC, Liao Y, Chang SH. Are Total and Domain -Specific Sedentary Time Associated with Overweight in Older Taiwanese Adults? Int J Environ Res Public Health 2015; 12(10):12697-12705.

11. Owen N, Sugiyama T, Eakin EE, Gardiner PA, Tremblay MS, Sallis JF. Adults' sedentary behavior determinants and interventions. Am J Prev Med 2011; 41(2):189-196.

12. Bauman A, Ainsworth BE, Sallis JF, Hagstromer M, Craig CL, Bull FC, Pratt M, Venugopal K, Chau J, Sjöström M, IPS Group. The descriptive epidemiology of sitting. A 20-country comparison using the International Physical Activity Questionnaire (IPAQ). Am J Prev Med 2011; 41(2):228-235.
13. O’Donoghue G, Perchoux C, Mensah K, Lakerveld J, van der Ploeg H, Bernaards C, Chastin SF, Simon C, O'Gorman D, Nazare JA, DEDIPAC Consortium. A systematic review of correlates of sedentary behaviour in adults aged 18-65 years: a socio-ecological approach. BMC Public Health 2016; 16:163.

14. Rhodes RE, Mark RS, Temmel CP. Adult sedentary behavior: a systematic review. Am J Prev Med 2012; 42(3):e3-28.

15. Gomes VB, Siqueira KS, Sichieri R. Physical activity in a probabilistic sample in the city of Rio de Janeiro. Cad Saude Publica 2001; 17(4):969-976.

16. Mielke GI, Silva IC, Owen N, Hallal PC. Brazilian adults' sedentary behaviors by life domain: population-based study. PLoS One 2014; 9(3):e91614.

17. Mielke GI, Hallal PC, Malta DC, Lee IM. Time trends of physical activity and television viewing time in Brazil: 2006-2012. Int J Behav Nutr Phys Act 2014; 11:101.

18. Suzuki CS, Moraes SA, Freitas IC. Sitting-time means and correlates in adults living in Ribeirao Preto-SP, Brazil, in 2006: OBEDIARP project. Rev Bras Epidemiol 2010; 13(4):699-712.

19. Instituto Brasileiro de Geografia e Estatística (IBGE). Censo Demográfico 2010 [página na Internet]. [acessado 2014 Jun 10]. Disponível em: http://censo2010. ibge.gov.br

20. Instituto Brasileiro de Geografia e Estatística (IBGE). Cidades@: São Leopoldo: RS [página na Internet]. 2016 [acessado 2014 set 30]. Disponível em: http:// www.ibge.gov.br/cidadesat/painel/painel.php?codmun $=432200$

21. Clemes SA, David BM, Zhao Y, Han X, Brown W. Validity of two self-report measures of sitting time. J Phys Act Health 2012; 9(4):533-539.

22. Healy GN, Clark BK, Winkler EA, Gardiner PA, Brown WJ, Matthews CE. Measurement of adults' sedentary time in population-based studies. Am J Prev Med 2011; 41(2):216-227.

23. Associação Brasileira de Empresas de Pesquisa (ABEP). Critério Brasil: Critério de classificação econômica 2015 [página na Internet]. [acessado 2014 ago 10]. Disponível em: http://www.abep.org/criterio-brasil

24. Costa JS, Silveira MF, Gazalle FK, Oliveira SS, Hallal PC, Menezes AM, Gigante DP, Olinto MT, Macedo S. Heavy alcohol consumption and associated factors: a population-based study. Rev Saude Publica 2004; 38(2):284-291.

25. Moreira LB, Fuchs FD, Moraes RS, Bredemeier M, Cardozo S, Fuchs SC, Victoria CG. Alcoholic beverage consumption and associated factors in Porto Alegre, a southern Brazilian city: a population-based survey. J Stud Alcohol 1996; 57(3):253-259.

26. Craig CL, Marshall AL, Sjostrom M, Bauman AE, Booth ML, Ainsworth BE, Pratt M, Ekelund U, Yngve A, Sallis JF, Oja P. International physical activity questionnaire: 12-country reliability and validity. Med Sci Sports Exerc 2003; 35(8):1381-1395.

27. Storgaard RL, Hansen HS, Aadahl M, Glumer C. Association between neighbourhood green space and sedentary leisure time in a Danish population. Scand $J$ Public Health 2013; 41(8):846-852. 
28. Barros AJ, Hirakata VN. Alternatives for logistic regression in cross-sectional studies: an empirical comparison of models that directly estimate the prevalence ratio. BMC Med Res Methodol 2003; 3:21.

29. Frank LD, Andresen MA, Schmid TL. Obesity relationships with community design, physical activity, and time spent in cars. Am J Prev Med 2004; 27(2):8796.

30. Koohsari MJ, Sugiyama T, Kaczynski AT, Owen N. Associations of leisure-time sitting in cars with neighborhood walkability. J Phys Act Health 2014; 11(6):1129-1132.

31. Wijndaele K, Sharp SJ, Wareham NJ, Brage S. Mortality Risk Reductions from Substituting Screen Time by Discretionary Activities. Med Sci Sports Exerc 2017; 49(6):1111-1119.

32. Del Pozo-Cruz J, Garcia-Hermoso A, Alfonso-Rosa RM, Alvarez-Barbosa F, Owen N, Chastin S, Del PozoCruz B. Replacing Sedentary Time: Meta-analysis of Objective-Assessment Studies. Am J Prev Med 2018; 55(3):395-402.

33. Bennie JA, Chau JY, van der Ploeg HP, Stamatakis E, Do A, Bauman A. The prevalence and correlates of sitting in European adults - a comparison of $32 \mathrm{Eu}-$ robarometer-participating countries. Int J Behav Nutr Phys Act 2013; 10:107.

34. Bennie JA, Pedisic Z, Timperio A, Crawford D, Duns$\tan$ D, Bauman A, van Uffelen J, Salmon J. Total and domain-specific sitting time among employees in desk-based work settings in Australia. Aust N Z J Public Health 2015; 39(3):237-242.

35. Pomerleau J, McKee M, Robertson A, Vaasc S, Kadziauskiene K, Abaravicius A, Bartkeviciute R, Pudule I, Grinberga D. Physical inactivity in the Baltic countries. Prev Med 2000; 31(6):665-672.

36. van der Ploeg HP, Venugopal K, Chau JY, van Poppel MN, Breedveld K, Merom D, Bauman AE. Non-occupational sedentary behaviors: population changes in The Netherlands, 1975-2005. Am J Prev Med 2013; 44(4):382-387.

37. Katzmarzyk PT, Church TS, Craig CL, Bouchard C. Sitting time and mortality from all causes, cardiovascular disease, and cancer. Med Sci Sports Exerc 2009; 41(5):998-1005.

38. Thraen-Borowski KM, Ellingson LD, Meyer JD, Cadmus-Bertram L. Nonworksite Interventions to Reduce Sedentary Behavior among Adults: A Systematic Review. Transl J Am Coll Sports Med 2017; 2(12):68-78.

39. Nooijen CFJ, Möller J, Forsell Y, Ekblom M, Galanti MR, Engström K. Do unfavourable alcohol, smoking, nutrition and physical activity predict sustained leisure time sedentary behaviour? A population-based cohort study. Prev Med 2017; 101:23-27.
40. Martin A, Fitzsimons C, Jepson R, Saunders DH, van der Ploeg HP, Teixeira PJ, Gray CM, Mutrie N, EuroFIT consortium. Interventions with potential to reduce sedentary time in adults: systematic review and meta-analysis. Br J Sports Med 2015; 49(16):1056-1063.

41. Saidj M, Menai M, Charreire H, Weber C, Enaux C, Aadahl M, Kesse-Guyot E, Hercberg S, Simon C, Oppert JM. Descriptive study of sedentary behaviours in 35,444 French working adults: cross-sectional findings from the ACTI-Cites study. BMC Public Health 2015; 15:379.

42. Oliveira AM, Miranda PR. Diferenças ocupacionais por raça e gênero no mercado de trabalho de trabalhadores no Brasil [Internet]. In: XI Encontro Nacional de Estudos Populacionais da Associação Brasileira de Empresas de Pesquisa 1998. [acessado 2018 Set 14]. Disponível em: http://www.abep.org.br/ abeporgb/ publicacoes/index.php/anais/article/viewFile/946/911

43. Wallmann-Sperlich B, Bucksch J, Schneider S, Froboese I. Socio-demographic, behavioural and cognitive correlates of work-related sitting time in German men and women. BMC Public Health 2014; 14:1259.

44. Chau JY, van der Ploeg HP, Merom D, Chey T, Bauman AE. Cross-sectional associations between occupational and leisure-time sitting, physical activity and obesity in working adults. Prev Med 2012; 54(34):195-200.

45. Jans MP, Proper KI, Hildebrandt VH. Sedentary behavior in Dutch workers: differences between occupations and business sectors. Am J Prev Med 2007; 33(6):450-454.

46. Hutcheson AK, Piazza AJ, Knowlden AP. Work Site -Based Environmental Interventions to Reduce Sedentary Behavior: A Systematic Review. Am J Health Promot 2018; 32(1):32-47.

47. Prince SA, Reed JL, McFetridge C, Tremblay MS, Reid RD. Correlates of sedentary behaviour in adults: a systematic review. Obes Rev 2017; 18(8):915-935.

48. Ku PW, Steptoe A, Liao Y, Hsueh MC, Chen LJ. A cut -off of daily sedentary time and all-cause mortality in adults: a meta-regression analysis involving more than 1 million participants. BMC Med 2018; 16:74.

Artigo apresentado em 14/07/2018

Aprovado em 05/11/2018

Versão final apresentada em 07/11/2018 